\title{
Prevalence of prelacteal feeding and its associated factors among mothers of under-24-month-old children at Arba Minch Zuria District, Ethiopia: A cross-sectional study
}

\author{
N D Megersa, ${ }^{1}$ MSc (Paed Nurs); E Z Tariku, ${ }^{2}$ MPH (Nutr); G E Yesera, ${ }^{1}$ MSc (Maternity Nurs); B T Gutema ${ }^{2,3}$ MSc (Nutr) \\ ${ }^{1}$ School of Nursing, College of Medicine and Health Sciences, Arba Minch University, Ethiopia \\ ${ }^{2}$ School of Public Health, College of Medicine and Health Sciences, Arba Minch University, Ethiopia \\ ${ }^{3}$ Arba Minch Health and Demographic Surveillance System, Ethiopia
}

Corresponding author: N Megersa (negshd05@gmail.com; nega.degefu@amu.edu)

\begin{abstract}
Background. Introduction of prelacteal feeds to newborn babies negates the recommendation of the World Health Organization that breastfeeding should be initiated within an hour after childbirth. As a result, many known health benefits of breastfeeding for infants, children and mothers are precluded. Therefore, to obviate the harmful effects of prelacteal feeding, it remains of paramount concern to identify the current status of prelacteal feeding and its contributing factors.

Objective. To assess the prevalence of prelacteal feeding and associated factors among mothers of under-24-month-old children in Arba Minch Zuria District, Ethiopia.

Methods. A community-based cross-sectional study was conducted among 400 mother/caregiver-child pairs. A structured and pretested questionnaire uploaded on mobile devices pre-installed with open data kit software was used for data collection. Factors associated with prelacteal feeding practices were explored using multivariable logistic regression analysis. The Hosmer-Lemeshow goodness-of-fit test was used to determine whether the model adequately described the data or not.

Results. A total of 400 mothers/caregivers participated in the study, of whom 67 (16.8\%) practised prelacteal feeding. Mothers who had poor knowledge of breastfeeding were nearly four times more likely to practise prelacteal feeding than those who had good knowledge (adjusted odds ratio (aOR) 3.95; 95\% confidence interval (CI) 1.82 - 8.54). Mothers who did not receive counselling on breastfeeding during antenatal care (ANC) follow-up were 4.1 times more likely to provide prelacteal feeds than those who received counselling (aOR 4.1; 95\% CI 1.70 - 9.76). Furthermore, mothers who did not receive immediate postnatal care were 6.46 times more likely to give prelacteal feeding than those who received immediate postnatal care (aOR 6.46; 95\% CI 2.85 - 14.63).

Conclusions. One out of six neonates was given prelacteal feeds in the study area. Poor knowledge among mothers about breastfeeding, lack of counselling on optimal breastfeeding during ANC visits, and lack of immediate postnatal care mainly led to prelacteal feeding. Therefore, attention should be given to improving maternal knowledge of breastfeeding through the refining of skilled counseling during ANC and immediate postnatal care.
\end{abstract}

S Afr J Child Health 2021;15(2):112-117. https://doi.org/10.7196/SAJCH.2021.v15i2.1698

Breastfeeding is an unmatched and most effective intervention to provide an ideal food for the growth and development of infants and young children. ${ }^{[1]}$ Breastfeeding has various known health benefits for infants, children and mothers. It also serves as a vital approach to improve public health at large; however, this can be achieved only when optimal breastfeeding can be implemented successfully. ${ }^{[2]}$ Optimal breastfeeding involves: initiation of breastfeeding in the first hour after birth, continued exclusively for the first six months of life ${ }^{[3]}$ and continued, with safe and adequate complementary foods, up to two years or beyond..$^{[2,4]}$

In contrast, the introduction of any fluids or foods other than breastmilk to a baby before the establishment of regular breastfeeding (prelacteal feeding) can deprive the child of valuable nutrients and colostrum, which thus puts the newborn at increased risk of infection. Besides, this malpractice has also been shown to delay the initiation of breastfeeding and hinders the proper establishment and future success of breastfeeding. ${ }^{[5]}$ Evidence suggests that providing prelacteal feeds can lead to early termination of full breastfeeding. ${ }^{[6]}$ Nevertheless, prelacteal feeds are given for non-nutritional purposes, in the belief that they can act as laxatives or cleansing or hydrating agents. ${ }^{[5]}$

Poor breastfeeding and complementary feeding practices are a global public concern. Worldwide, it is estimated that only $35 \%$ of infants are exclusively breastfed for the first six months of life, while the majority of infants receive some other food or fluid in their early months. ${ }^{[7]}$

Developing countries share the greatest burden of death related to suboptimal breastfeeding, especially non-exclusive breastfeeding in the first six months of life, which results in 1.4 million deaths and $10 \%$ of the disease burden in children under five years. ${ }^{[1]}$ If promotion and support for early initiation and exclusive breastfeeding can be made universal, there would be a $45 \%$ reduction in all-cause and infection-related neonatal mortality, also considering that about $12 \%$ of deaths of children under two years have been attributed to suboptimal breastfeeding. ${ }^{[8]}$

The prevalence of prelacteal feeding in sub-Saharan Africa has been calculated to be $32.2 \%$. Plain water, milk other than breastmilk, 
and sugar or glucose water were some of the prelacteal feeds given to babies. ${ }^{[9]}$ A wide range of harmful newborn feeding practices including prelacteal feeding has been documented in Ethiopia, even after the implementation of infant and young child feeding programmes. The findings of the recent nationwide Ethiopian Demographic and Health Survey (EDHS) showed that the prevalence of prelacteal feeding was $8 \% .{ }^{[10]}$

There is a variation in the prevalence of prelacteal feeding from place to place within the country, $75.5 \%$ in the Kersa District, ${ }^{[11]}$ $45.4 \%$ in the Harari region, ${ }^{[12]} 42.9 \%$ in $\mathrm{Afar}^{[13]} 38.8 \%$ in Raya Kobo $^{[14]}$ and $15 \%$ in Bahir Dar city. ${ }^{[15]}$

Studies so far have identified different factors contributing to prelacteal feeding. These include: father's educational status, mother's occupation, average monthly household income, ${ }^{[13]}$ time of initiation of breastfeeding, ${ }^{[12-14]}$ mode of delivery, ${ }^{[14]}$ place of delivery ${ }^{[12,14]}$ and antenatal care (ANC) follow-up. ${ }^{[12]}$

Many aspects of the relationship between prelacteal feeding and maternal sociodemographic characteristics and healthcare utilisation have been extensively studied and described in the literature. However, there is a paucity of evidence on how equally important factors such as immediate postnatal care (PNC) and maternal breastfeeding knowledge affect prelacteal feeding practice, and were given little attention, particularly in the study area. The present study was therefore aimed to assess the prevalence of prelacteal feeding and its associated factors among mothers of under-24-month-old children in Arba Minch Zuria District, Ethiopia.

\section{Methods and materials Study design and setting}

A community-based cross-sectional study was conducted from February to March 2018 in Arba Minch Health and Demographic Surveillance System (HDSS) sites which were hosted by Arba Minch University. The sites are located in the Arba Minch Zuria District in southern Ethiopia. According to the HDSS report, the district has a total population of 74157 , of whom 3521 are children under two years. The district comprises 31 kebeles (wards) of which, nine kebeles were within the HDSS limit. The area has three climatic zones: highland, midland and lowland.

\section{Sample size determination and sampling procedure}

The sample size was calculated using the single population proportion formula by considering the following assumption: $95 \%$ confidence interval (CI), $5 \%$ margin of error, $10 \%$ for non-response, and best estimates of the proportion of mothers who gave prelacteal feeding being $38.8 \%$ from the previous study at Raya Kobo. ${ }^{[14]}$ Accordingly, the final computed sample size was 402 .

All kebeles within the limits of Arba Minch HDSS were included in the study. Hence, all mothers of under-24-month-old children residing within these kebeles were our source population. The list of mothers with a child under the age of 24 months in these kebeles was retrieved from the HDSS database using the mothers' identification numbers. Then a simple random sampling method was used to select study participants by generating a table of random numbers using Stata statistical software (StataCorp., USA). Finally, the randomly selected participants were accessed in their dwellings by referring to their HDSS household ID.

\section{Data collection tool and procedure}

Data were collected using a structured and pretested questionnaire which was adapted from prior studies conducted in different areas and modified accordingly. ${ }^{[12,14-16]}$ The questionnaire contained items on sociodemographic characteristics, pregnancy, childbirth, early infant feeding practices, and mothers' health services utilisation. The questionnaire was uploaded on mobile devices running an Android operating system which was preinstalled with Open Data Kit (ODK; Nafundi, USA)) data collection software. In total, nine trained and experienced data collectors and three field supervisors working in Arba Minch HDSS sites collected the data using hand-held devices. The data collectors visited 13 households per day. The collected data were then sent to a server in the HDSS office, aggregated and downloaded. Training was given for two days to data collectors and supervisors, and mainly focused on data collection tools and the mobile data collection platform using ODK to equip data collectors with the skills necessary to manage data collection. The questionnaire was thoroughly reviewed by a senior expert who had experience in question construction to make sure the questionnaire had no mistakes. An expert familiar with the topic also reviewed the tool to see if the questions captured the topic well. A pretest was also conducted on 20 mothers living in a kebele not enrolled in the study, to ensure the quality of the data as well. Data from the pretest were excluded after correcting the wording, sequence and approach of the questions.

\section{Data processing and analysis}

The collected data were cleaned, coded and checked for missing values, and then analysed using SPSS version 20 software (IBM Corp., USA). Descriptive statistics such as frequencies, percentages and proportions were used to summarise pertinent variables and presented using tables and graphs. The family wealth index was constructed via the principal component analysis (PCA) method and the family wealth status was grouped into quantiles. A binary logistic regression model was used to identify factors associated with prelacteal feeding. Crude odds ratios (cOR) with their 95\% CIs were computed to assess the association between each independent variable and the outcome variable and to identify variables that were candidates for multivariable analysis. Variables with a $p$-value $<0.25$ in bivariate logistic regression were entered into the multivariable analysis. An adjusted odds ratio (aOR) with a $95 \% \mathrm{CI}$ was used to assess the strength of association, and a $p$-value $<0.05$ was used to assert statistical significance in the final model. The collinearity effect was tested using the variance inflation factor (VIF). Model fitness was checked by using the Hosmer and Lemeshow goodnessof-fit test with the enter method.

\section{Measurements and operational definitions}

Prelacteal feeding is defined as feeding newborns anything other than breastmilk before breastfeeding is initiated or regularly given within the first three days from birth. ${ }^{[10]}$ This was assessed by asking mothers a single question: 'Did you give any food/fluid to your baby in the first three days after birth?' A dichotomised possible response of Yes was scored as 1 and No scored as 0.

Index child is the last child of the mother who was less than two years old and for whom the feeding practice was asked.

Maternal breastfeeding knowledge was assessed by mothers' responses to the following three questions related to early breastfeeding: (i) How long after birth should a newborn start breastfeeding? (correct response: Immediately or less than one hour after delivery.); (ii) What do you do with colostrum? (correct response: Give to the infant.); (iii) How long should the baby breastfeed exclusively? (correct response: 6 months). Each question was given a score of 1 or 0 , depending on a correct or incorrect response, respectively. We then created an overall score of correct knowledge response of $0-3$. Thus, those who responded to all three questions correctly had good knowledge, those 
with two correct responses had moderate knowledge, and those with one or no correct response had poor knowledge.

\section{Results}

\section{Sociodemographic characteristics}

A total of 400 mother-child pairs participated in the study, yielding a response rate of $99.5 \%$. The mean (standard deviation (SD)) age of study participants was 29.01 (5.39) and ranged from 16 to 42 years. Nearly $77 \%$ of study participants were followers of the Protestant religion. The majority of study participants (326 (81.5\%)) were of Gamo ethnicity. Seventy-six percent of all mothers in the study area were housewives by occupation. The mean (SD) age of the index child was 9.02 (6.99) months (Table 1).

Table 1. Sociodemographic characteristics of mothers of under-24-month-old children in Arba Minch Zuria District, Ethiopia, in 2018

\begin{tabular}{ll}
\hline Characteristics & $n(\%)$ \\
\hline Age, years & \\
$16-24$ & $92(23)$ \\
$25-34$ & $231(57.8)$ \\
$\geq 35$ & $77(19.3)$ \\
Ethnicity & \\
Gamo & $326(81.5)$ \\
Zeise & $54(13.5)$ \\
Other & $20(5.1)$ \\
Religion & \\
Protestant & $309(77.3)$ \\
Orthodox Christian & $86(21.5)$ \\
Other & $5(1.3)$ \\
Occupation & \\
Housewife & $304(76.8)$ \\
Farmer & $64(16.0)$ \\
Other & \\
Marital status & $29(7.2)$ \\
Married & \\
Never married & $393(98.3)$ \\
Separated/widowed & $1(0.3)$ \\
Educational status & $6(1.5)$ \\
No formal education & \\
Formal education & \\
Child's sex & $232(58.0)$ \\
Male & $168(42.0)$ \\
Female & \\
Child's age (months) & $205(51.2)$ \\
0 - 11 & $195(48.8)$ \\
$12-23$ & \\
Husband's educational status $(n=393)$ & $244(61.0)$ \\
No formal education & $156(39.0)$ \\
Formal education & \\
Lowily wealth status & $190(48.3)$ \\
Middle & $134(33.5)$ \\
High & \\
& \\
&
\end{tabular}

Maternal health care utilisation-related characteristics

From the total of 400 mothers, 328 (82\%) had ANC visits, of whom $49 \%$ had four or more visits. Only about one-third (32.9\%) of them received counselling on breastfeeding. Nearly one-third of all mothers received immediate PNC. More than half (51.9\%) of all mothers gave birth to an index child at home and almost all (97\%) of the mothers delivered their index child spontaneously via the vagina. The majority of study participants (80.5\%) had more than one birth (were multiparous) (Table 2).

\section{Prevalence of prelacteal feeding}

Out of 400 mothers, prelacteal feeding was given by $16.8 \%$ (95\% CI 13.0 - 20.0). Of the latter, non-breastmilk constituted the most common prelacteal feed, and was given by $42(10.5 \%)$ of the respondents. Other prelacteal feeds were sugar water and coffee leaves boiled in sugar water. Of those who gave prelacteal feeds, $13.5 \%$ said that insufficiency of breastmilk was a major reason for providing prelacteal feeds (Table 3 ).

\section{Mothers' knowledge of breastfeeding}

Most mothers (85.8\%) knew that breastfeeding should begin immediately or within one hour after birth, and $53.5 \%$ of mothers

\begin{tabular}{|c|c|}
\hline Variables & $n(\%)$ \\
\hline \multicolumn{2}{|c|}{ ANC follow-up for the index child $(n=400)$} \\
\hline Yes & $328(82.0)$ \\
\hline No & $72(18.0)$ \\
\hline \multicolumn{2}{|c|}{ Number of ANC follow-ups $(n=328)$} \\
\hline 1 & $4(1.0)$ \\
\hline $2-3$ & $127(31.8)$ \\
\hline$\geq 4$ & $197(49.3)$ \\
\hline \multicolumn{2}{|c|}{$\begin{array}{l}\text { Received counselling on breastfeeding at ANC } \\
(n=328)\end{array}$} \\
\hline Yes & $108(32.9)$ \\
\hline No & $220(67.1)$ \\
\hline \multicolumn{2}{|l|}{ Place of delivery } \\
\hline Home & $192(48.0)$ \\
\hline Health facility & $208(52.0)$ \\
\hline \multicolumn{2}{|l|}{ Mode of delivery } \\
\hline Caesarean & $12(3.0)$ \\
\hline Vaginal & $388(97.0)$ \\
\hline \multicolumn{2}{|c|}{ Perceived size of baby at birth } \\
\hline Small & $121(30.3)$ \\
\hline Medium & $212(53.0)$ \\
\hline Large & $67(16.8)$ \\
\hline \multicolumn{2}{|c|}{ Immediate postnatal care } \\
\hline Yes & $135(33.8)$ \\
\hline No & $265(66.3)$ \\
\hline \multicolumn{2}{|c|}{$\begin{array}{l}\text { Encountered breast problem after delivery of index } \\
\text { child }\end{array}$} \\
\hline Yes & $60(15.0)$ \\
\hline No & $340(85.0)$ \\
\hline \multicolumn{2}{|l|}{ Parity } \\
\hline Primipara & $78(19.5)$ \\
\hline Multipara & $322(80.5)$ \\
\hline
\end{tabular}


Table 3. Prelacteal feeding practices of mothers of under24-month-old children in Arba Minch Zuria District, Ethiopia, in 2018

\begin{tabular}{ll}
\hline Feeding practices & $n(\%)$ \\
\hline $\begin{array}{l}\text { Did you give any food/fluid to your newborn before } \\
\text { the first } 3 \text { days of life? }\end{array}$ \\
$\begin{array}{l}\text { No } \\
\text { Yes }\end{array}$ & $333(83.3)$ \\
Type of prelacteal food given to baby & $67(16.8)$ \\
Milk other than breastmilk & \\
Rolled butter & $42(10.5)$ \\
Clean water & $7(1.8)$ \\
Other & $3(0.8)$ \\
Reason for giving prelacteal food $(n=67)$ & $15(3.8)$ \\
Insufficient breastmilk & \\
To stimulate sucking & $54(13.4)$ \\
For cleaning infants' GIS & $2(0.5)$ \\
Other & $4(1.0)$ \\
Influence from others to give prelacteal food $(n=67)$ & $7(1.8)$ \\
No & $36(46.3)$ \\
Yes & $36(53.7)$ \\
Source of influence to give prelacteal food $(n=36)$ & \\
Family & $12(33.3)$ \\
Neighbour & $16(44.4)$ \\
TBA & $8(22.2)$
\end{tabular}

GIS = gastro-intestinal system; TBA = traditional birth attendant.

recognised that babies should be fed colostrum. Nearly two-thirds $(68.5 \%)$ of study participants mentioned that a child should be fed only breastmilk until six months of age. Overall, $46.0 \%, 17.8 \%$ and $36.3 \%$ of all study participants had good, moderate and poor knowledge, respectively, of breastfeeding.

\section{Factors associated with prelacteal feeding practice}

In the multivariable model, not receiving counselling on breastfeeding, lack of immediate PNC, and poor mothers' knowledge of breastfeeding were positively associated with prelacteal feeding $(p<0.05)$. Mothers who did not receive counselling about breastfeeding during antenatal follow-up were 4.1 times more likely to provide prelacteal feed to their babies, compared with those who received counselling ( $\mathrm{aOR} 4.1 ; 95 \% \mathrm{CI} 1.70$ - 9.76). The odds of giving prelacteal feed were 6.46 times higher among mothers who did not have immediate postnatal care than those who received immediate PNC (aOR 6.46; 95\% CI 2.85 - 14.63). Furthermore, mothers who had a poor and moderate level of knowledge about breastfeeding were 3.95 and 3.12 times more likely to give prelacteal feeds to their babies than those who had good knowledge, respectively. Hence, as mothers' knowledge of of breastfeeding increases, prelacteal feeding practices decrease (Table 4).

\section{Discussion}

Considerable improvements have been achieved in prelacteal feeding practices in Ethiopia. A countrywide survey showed a reduction in the prevalence of prelacteal feeding from $27 \%$ in $2011^{[17]}$ to $8 \%$ in 2016. ${ }^{[10]}$ However, in the current study, the prevalence of prelacteal feeding was $16.8 \%$, which is two times higher than stated in the recent EDHS report. The prevalence of prelacteal feeding in the current study is also higher than that in a finding in North Wello Zone of $11.1 \%{ }^{[16]}$ This difference may be attributed to disparity in the residency of respondents. In the latter study, participants resided in cities where there was broader access to information and healthcare services, unlike the current study which was conducted in rural parts of the district. A nearly consistent finding was reported in a study from Bahir Dar City, in which the prevalence of prelacteal feeding was $15 \%{ }^{[15]}$

The prevalence of prelacteal feeding in the present study was significantly lower than that reported in Jammu and Kashmir in India (88\%), ${ }^{[18]}$ Kersa District in Ethiopia $(75.8 \%),{ }^{[1]}$ and Vietnam (73.3\%). ${ }^{[19]}$ This substantial variance may be credited to differences in cultural beliefs, practices related to newborn feeding, and sample size. Additionally, higher prevalences of prelacteal feeding have been reported in Egypt (57.8\%) ${ }^{[20]}$ and the Afar (42.9\%), ${ }^{[13]}$ Harar $(45.4 \%)^{[12]}$ and Raya Kobo regions (38.8\%). ${ }^{[14]}$

The finding of a study from northern Nigeria also showed a significantly higher prevalence $(85.2 \%)$ of prelacteal feeding, as compared with the current finding. ${ }^{[21]}$ This discrepancy might result from a difference in the respondents' age category. In the former study, participants were younger girls aged 10 - 14 years who were married. This group of younger girls was liable to give prelacteal feeds because they were not sufficiently mature and experienced to feed newborns.

Our study revealed that milk other than mothers' breastmilk accounted for the highest proportion (10.5\%) of prelacteal feeds provided by the mother, followed by rolled butter (1.8\%) and clean water $(0.8 \%)$. Butter was also reported as a common prelacteal feed in Raya Kobo ${ }^{[14]}$ whereas plain water was described as a predominant prelacteal feed in the Harari region. ${ }^{[12]}$

In the current study, counselling on breastfeeding during ANC visits was associated with reduced risk of prelacteal feeding practice. A consistent finding was reported in a study from Kolkata and Maharashtra in India, and in north-eastern Ethiopia, in which the odds of giving prelacteal feeds were higher among mothers who had not received counselling than among those who had. ${ }^{[16,22,23]}$

The present study found that mothers who had received immediate PNC had a lower likelihood of giving prelacteal feeding than those who had not received it; this was because mothers who were visited by healthcare providers immediately after birth (within six hours) would have a greater chance of obtaining counselling on optimal breastfeeding.

A lower level of knowledge about breastfeeding was one of the significant factors that was positively associated with prelacteal feeding practice in our study. A parallel finding was reported in studies from rural Sidama and Vietnam in which a higher knowledge score on breastfeeding corresponds with minimal odds of giving prelacteal feeds. ${ }^{[19,24]}$ This evidence signals an increasing need for encouraging strategies that enhance the dissemination of health information to improve knowledge of the community about optimal breastfeeding.

The main strength of this study is the utilisation of an appropriate model for analysis and sampling procedure to yield a representative sample. Despite this strength, the study also had the following shortcomings: its findings rely on the women's memory, which is subjected to recall bias, even though attempts such as probing to help them remember, were made. The study also has the drawbacks of cross-sectional study design.

\section{Conclusions and recommendations}

Despite the implementation of infant and young child feeding programmes, one out of six neonates in the study area still receives prelacteal feeds. Prelacteal feeding practice thrives among mothers who do not have immediate postnatal care, those who did not receive counselling about breastfeeding during ANC visits, and mothers 
Table 4. Factors associated with prelacteal feeding among mothers of under-24-month-old children in Arba Minch Zuria District, Ethiopia, in 2018

\begin{tabular}{|c|c|c|c|c|}
\hline \multirow[b]{2}{*}{ Factors } & \multicolumn{2}{|c|}{ Prelacteal feeding } & \multirow[b]{2}{*}{ cOR $(95 \% \mathrm{CI})$} & \multirow[b]{2}{*}{ aOR $(95 \% \mathrm{CI})$} \\
\hline & No, $n(\%)$ & Yes, $n(\%)$ & & \\
\hline \multicolumn{5}{|l|}{ Mother's educational level } \\
\hline No formal education & $201(86.6)$ & $31(13.4)$ & $0.57(0.33-0.96)^{*}$ & $0.58(0.29-1.17)$ \\
\hline Formal education & $132(78.6)$ & $36(21.4)$ & 1 & 1 \\
\hline \multicolumn{5}{|c|}{ Received counselling on BF } \\
\hline No & $167(75.9)$ & $53(24.1)$ & $3.97(1.81-8.69)^{*}$ & $4.1(1.70-9.76)^{\star}$ \\
\hline Yes & $100(92.6)$ & $8(7.4)$ & 1 & 1 \\
\hline \multicolumn{5}{|c|}{ Breast problem encountered } \\
\hline No & $287(84.4)$ & $53(15.6)$ & 1 & 1 \\
\hline Yes & $46(76.7)$ & $14(23.3)$ & $0.61(0.31-1.18)$ & $1.46(0.63-3.39)$ \\
\hline \multicolumn{5}{|c|}{ Perceived size of baby at birth } \\
\hline Small & $96(79.3)$ & $25(20.7)$ & $0.90(0.44-1.86)$ & $1.58(0.62-4.05)$ \\
\hline Medium & $185(87.3)$ & $27(12.7)$ & $0.51(0.25-1.02)$ & $0.50(0.22-1.18)$ \\
\hline Large & $52(77.6)$ & $15(22.4)$ & 1 & 1 \\
\hline \multicolumn{5}{|l|}{ Place of delivery } \\
\hline Home & $165(85.9)$ & $27(14.1)$ & $0.68(0.40-1.17)$ & $0.72(0.35-1.49)$ \\
\hline Health facility & $168(80.8)$ & $40(19.2)$ & 1 & 1 \\
\hline \multicolumn{5}{|l|}{ Immediate postnatal care } \\
\hline No & $208(78.5)$ & $57(21.5)$ & $3.43(1.69-6.95)$ & $6.46(2.85-14.63)^{\star}$ \\
\hline Yes & $125(92.6)$ & $10(7.4)$ & 1 & 1 \\
\hline \multicolumn{5}{|c|}{ Mother's knowledge of BF } \\
\hline Poor & $114(78.6)$ & $31(21.4)$ & $2.11(1.15-3.86)^{*}$ & $3.95(1.82-8.54)^{\star}$ \\
\hline Moderate & $56(78.9)$ & $15(21.1)$ & $2.08(1.00-4.31)^{*}$ & $3.12(1.26-7.72)^{*}$ \\
\hline Good & $163(88.6)$ & $21(11.4)$ & 1 & 1 \\
\hline \multicolumn{5}{|l|}{ Family wealth status } \\
\hline Low & $103(77.4)$ & $30(22.6)$ & $2.48(1.25-4.92)^{*}$ & $2.18(0.95-4.99)$ \\
\hline Middle & $111(82.8)$ & $23(17.2)$ & $1.76(0.86-3.59)$ & $1.41(0.59-3.38)$ \\
\hline High & $333(89.5)$ & $14(10.5)$ & 1 & 1 \\
\hline
\end{tabular}

with poor knowledge of breastfeeding. The findings of this study, therefore, emphasise the need to improve mothers' knowledge of breastfeeding, and the importance of skilled counselling on optimal breastfeeding during ANC visits. Furthermore, special attention should be given to mothers during immediate postnatal care to mitigate the burden of prelacteal feeding practice. Lastly, this study calls for further qualitative research in the study area to anticipate how best the finding of this study will be utilised.

Declaration. An institutional review board of Arba Minch University College of Medicine and Health Science approved this study. An official letter from Arba Minch University College of Medicine and Health Sciences research co-ordination office was submitted to the zonal health office. A permission letter from the zone was submitted to the Arba Minch Zuria District authority. Verbal informed consent was obtained from study participants before commencing face-to-face interviews.

Acknowledgements. First and foremost, we express our gratitude to Arba Minch University College of Medicine and Health Sciences for the opportunity to conduct this research. Our special thanks go to the Arba Minch HDSS Centre for providing preliminary data and the sampling frame. The authors are also glad to affirm their appreciation of the data collectors, supervisors and various stakeholders who helped us during data collection.

Author contributions. ND, EZ and GE participated in conceiving the study and wrote the proposal. BT and ND supervised the data collection process and reviewed the manuscript frequently. ND, EZ, BT and GE were also involved in the method, analysis and interpretation of the findings. The datasets used and/or analysed during the current study are available from the corresponding author on request.

Funding. This study was supported by Arba Minch University.

Conflicts of interest. None.

1. World Health Organization. Infant and young child feeding: Model chapter for textbooks for medical students and allied health professionals. Geneva: WHO, 2009

2. United Nations Children's Emergency Fund. Nurturing the health and wealth of nations: The investment case for breastfeeding. New York: UNICEF, 2017:12. (accessed 21 February 2017).

3. Kramer MS, Kakuma R. The optimal duration of exclusive breastfeeding. Protecting infants through human milk. New York: Springer, 2004:63-77.

4. World Health Organization. Guiding principles for feeding non-breastfed children 6 - 24 months of age. Geneva: WHO, 2005.

5. Laroia N, Sharma D. The religious and cultural bases for breastfeeding practices among the Hindus. Breastfeed Med 2006;1(2):94-98. https://doi.org/10.1089/ bfm.2006.1.94 
6. Lakati A, Makokha O, Binns C, Kombe Y. The effect of pre-lacteal feeding on full breastfeeding in Nairobi, Kenya. East Afr J Publ Health 2011;7(3):258-262.

7. World Health Organization. Global data bank on infant and young child feeding. Brasília: WHO, 2009.

8. World Health Organization. The health of the people: What works: The African Regional Health Report 2014. Geneva: WHO, 2014.

9. Berde AS, Ozcebe H. Risk factors for prelacteal feeding in sub-Saharan Africa: A multilevel analysis of population data from twenty-two countries. Public Health Nutr 2017;20(11):1953-1962. https://doi.org/10.1017/S1368980017000659

10. Central Statistical Agency - CSA/Ethiopia, ICF. Ethiopia Demographic and Health Survey 2016. Addis Ababa: CSA and ICF, 2017.

11. Egata G, Berhane Y, Worku A. Predictors of non-exclusive breastfeeding at 6 months among rural mothers in east Ethiopia: A community-based analytical cross-sectional study. Int Breastfeed J 2013;8(1):8. https://doi. org/10.1186/1746-4358-8-8

12. Bekele Y, Mengistie B, Mesfine F. Prelacteal feeding practice and associated factors among mothers attending immunisation clinic in Harari Region public health facilities, Eastern Ethiopia. Open J Prevent Med 2014;4(7):529-534. https://doi.org/10.4236/ojpm.2014.47063

13. Liben ML, Wuneh AG, Zepro NB, Mulugeta A. Factors associated with prelacteal feeding in Afar regional state, northeastern Ethiopia: A cross sectional study. Int J Res 2017;5(7):116-127. https://doi.org/10.29121/ granthaalayah.v5,i7.2017.2112

14. Legesse M, Demena M, Mesfin F, Haile D. Prelacteal feeding practices and associated factors among mothers of children aged less than 24 months in Raya Kobo district, North Eastern Ethiopia: A cross-sectional study. Int Breastfeed J 2014;9(1):189. https://doi.org/10.1186/s13006-014-0025-2

15. Demilew YM, Tafere TE, Abitew DB. Infant and young child feeding practice among mothers with 0-24 months old children in slum areas of Bahir Dar City, Ethiopia. Int Breastfeed J 2017;12:26. https://doi.org/10.1186/s13006-017-0117-X
16. Bililign N, Kumsa H, Mulugeta M, Sisay Y. Factors associated with prelacteal feeding in North Eastern Ethiopia: A community based cross-sectional study. Int Breastfeed J 2016;11:13. https://doi.org/10.1186/s13006-016-0073-x

17. Central Statistical Agency of Ethiopia, ICF International. Ethiopia Demographic and Health Survey 2011. Addis Ababa: Central Statistical Agency/Ethiopia and ICF International, 2012.

18. Raina SK, Mengi V, Singh G. Determinants of prelacteal feeding among infants of RS Pura block of Jammu and Kashmir, India. J Family Med Prim Care 2012;1(1):27.

19. Nguyen PH, Keithly SC, Nguyen NT, Nguyen TT, Tran LM, Hajeebhoy N. Prelacteal feeding practices in Vietnam: Challenges and associated factors. BMC Public Health 2013;13:932. https://doi.org/10.1186/1471-2458-13-932

20. El-Gilany AH, Abdel-Hady DM. Newborn first feed and prelacteal feeds in Mansoura, Egypt. Biomed Res Int 2014:7. https://doi.org/10.1155/2014/258470

21. Jimoh AO, Adaji SE, Adelaiye HA, et al. Factors associated with prelacteal feeding practices in a rural northern Nigerian setting. S Afr J Clin Nutr 2017;31(2):37-42. https://doi.org/10.1080/16070658.2017.1359391

22. Roy S, Dasgupta A, Pal B. Feeding practices of children in an urban slum of Kolkata. Indian J Community Med 2009;34(4):362. https://doi. org/10.4103\%2F0970-0218.58402

23. Dawal S, Inamdar I, Saleem T, Priyanka S, Doibale M. Study of pre lacteal feeding practices and its determinants in a rural area of Maharashtra. Sch J App Med Sci 2014;2(4):1422-1427.

24. Chea N, Asefa A. Prelacteal feeding and associated factors among newborns in rural Sidama, south Ethiopia: A community based cross-sectional survey. Int Breastfeed J 2018;13:7. https://doi.org/10.1186/s13006-018-0149-x

Accepted 28 November 2020. 